\title{
Reconfiguration of distribution network with DG based on Double perturbation mutation particle swarm optimization
}

\author{
Lan Zhang ${ }^{1,}$, Jian Zhang ${ }^{1, b}$ \\ ${ }^{1}$ School of Electrical Engineering, Zhengzhou University, Zhengzhou 450001, China. \\ azhanglan_0827@163.com, b zhangjian63@zzu.edu.cn
}

\begin{abstract}
Keywords: Gauss white noise, disturbance variation, distributed generation, distribution network reconfiguration.
\end{abstract}

Abstract. Due to the rise of distributed generation, it is very important to consider the impact of distributed generation in distribution network reconfiguration. To solve the problem, this paper uses the double perturbation mutation particle swarm optimization algorithm, which is to introduce the Gauss white noise disturbance into the particle swarm algorithm and introduce inertia weight of the dynamic change and sigmoid function to avoid the premature convergence. Through the analysis of the example, the results prove the feasibility of the improved algorithm, and indicate that appropriate position of distributed generator can reduce the network losses and improve the voltage.

\section{Introduction}

Distribution network reconfiguration, which can reduce the network loss and improve the voltage quality and power system reliability , is to change the topology of the network by opening and closing some branch switches or contact switches[1]. Due to the energy and environmental problems, in recent years distributed generation with so many advantages, such as high utilization rate of the new energy, low investment cost and running flexible, has been applied widely in electrical power system. So considering the distributed generation in the reconfiguration of distribution network is badly needed.

Reconstruction problem is a nonlinear multi-dimensional optimization problem [2], scholars have put forward lots of algorithm, in which the particle swarm algorithm is relatively simple, and of fast convergence, but it's easy to fall into local optimum. In Ref. [3], the particle swarm optimization and multi Agent intelligent search are combined to jump out of local optimum. In Ref. [4], the tabu search algorithm is combined with the particle swarm optimization algorithm, regarding the distributed generation with fixed position and fixed capacity as the negative PQ node. In Ref. [5], ant colony algorithm is used to determine the capacity and location of distributed generation. In this paper, the Gauss white noise perturbation and genetic variation and the dynamic change of inertia weight are introduced into the particle swarm optimization algorithm to avoid falling into local optimum. And the reconstruction in this paper considers both the network reconfiguration and the influence of the location of the distributed generation. Through analysis by IEEE33 node network, the results show that the algorithm is feasible, and can get rid of the shortcomings of local optimum.

\section{Mathematical Model}

Distribution network reconfiguration optimization has many goals, such as raising voltage level, minimizing loss, running the best benefits, etc. This paper uses the minimum active power loss as the objective function [2], the expression is as shown in Eq.1:

$$
\min F=\sum_{j=1}^{n} R_{j} \frac{P_{j}^{2}+Q_{j}^{2}}{U_{j}^{2}}
$$

In the above formula, $F$ is the active network loss, $n$ is the total number of branches with closed switches, $P_{j} 、 Q_{j}$ are respectively the active and reactive power flowing on the branch $j, V_{j}$ is the node voltage at the end of the branch $j$.

In addition, some constraints should also be satisfied: 
Node voltage constraint is shown in Eq.2.

$$
U_{i \min } \leq U_{i} \leq U_{i \max }
$$

In Eq.2, $U_{i \min } 、 U_{i \max }$ are respectively the maximum and minimum allowable voltage of node $i$.

Capability constraint of branches is shown in Eq.3.

$$
I_{j} \leq I_{j \max }
$$

In Eq.3, $I_{j \max }$ is the maximum allowable current for branch $j$.

Power flow constraint is shown in Eq.4 and 5.

$$
\begin{aligned}
& P_{i}+P_{D G}>0 \\
& Q_{i}+Q_{D G}>0
\end{aligned}
$$

In Eq.4 and 5, $P_{i} 、 Q_{i} 、 P_{D G} 、 Q_{D G}$ are respectively the active power and reactive power flowing through the node $i$ and capacity of the distributed generation of the node $i$.

The reconstructed network structure must still be radiation network, with no loop and no island. All points are connected and the total number of closed branches equals to the number of nodes minus 1 .

\section{Double Perturbed Particle Swarm Optimization Algorithm}

Basic particle swarm optimization algorithm. Particle swarm optimization (PSO), which originated from foraging behavior of birds, is a biological intelligent optimization algorithm. Starting from random solutions, each solution is a particle, and each particle has its position and velocity and the corresponding fitness function value, iterating formula of the speed and position. In each iteration, the formula updates through the two values, of which, the first one is called the individual extreme value that is the optimal particle itself to find solutions, the second is called the global optimal solution, which is the optimal solution of the whole population [6]. Its velocity and position updating equations are shown in Eq.6 and Eq.7.:

$$
\begin{gathered}
v_{i d}^{k+1}=w v_{i d}^{k}+c_{1} r_{1}\left(p_{i d}^{k}-x_{i d}^{k}\right)+c_{2} r_{2}\left(p_{g d}^{k}-x_{i d}^{k}\right) \\
x_{i d}^{k+1}=x_{i d}^{k}+v_{i d}^{k+1}
\end{gathered}
$$

In Eq.6 and Eq.7, $v_{i d}^{k} 、 v_{i d}^{k+1} 、 x_{i d}^{k} 、 x_{i d}^{k+1}$ are respectively the speed and position of the particle $i$ on its $d$ dimension when the program iterates $k 、 k+1$ times; $w$ is the inertia weight to show the effects of particle's history velocity on current velocity in the iterative process; $c_{1} 、 c_{2}$ are called acceleration factors or learning factor, which represent the particle's individual cognitive and social experience; $r_{1}$ 、 $r_{2}$ are random numbers in $[0,1] ; p_{i d}^{k} 、 p_{g d}^{k}$ are respectively individual extremum and global extremum.

Encoding style. The distribution network is the radiation network, and its topology determines that the disconnected branch number (branch number minus node number plus 1) is fixed, which can avoid some unreasonable solution. Therefore in the encoding, if broken $n$ branches, each particle encodes with $n$ dimensions to express disconnected switches. If there are $m$ DG in the system, then every particle has $(m+n)$ dimensions. This encoding method is more convenient and only need two parameter matrices, namely the node matrix and branch matrix, to run the program.

Improving velocity formula. In particle swarm optimization, inertia weight has a significant impact on the performance of the algorithm. We can use $w$ with a smaller values to improve the local search ability of the algorithm, or can use $w$ with a larger value to improve the algorithm's global search ability, so choosing a appropriate inertia weight can improve the search efficiency and avoid falling into local optimum. This paper introduces a dynamic inertia weight like Eq.8.

$$
w(k)=\left(w_{i n i}-w_{\text {end }}\right) \arctan \frac{K_{\max }-k}{K_{\max }}
$$


In Eq.8, $w(k)$ is the inertia weight when the algorithm iterates $k$ times. $w_{\text {ini }}$ is the initial inertia weight and $w_{\text {end }}$ is the final inertia weight. $K_{\max }$ is the maximum number of iterations.

After introducing dynamic inertia weight, the velocity formula becomes like Eq.9.

$$
v_{i d}^{k+1}=w(k) v_{i d}^{k}+c_{1} r_{1}\left(p_{i d}^{k}-x_{i d}^{k}\right)+c_{2} r_{2}\left(p_{g d}^{k}-x_{i d}^{k}\right)
$$

In addition, because of integer coding, velocity formulas need to be improved. The Sigmoid function is introduced to modify the velocity equation as shown in Eq.10.

$$
v_{i d}^{k+1}=\operatorname{round}\left(\left(1 /\left(1+\exp \left(-v_{i d}^{k+1}\right)\right)\right) \times \text { randn } \times \text { range }\right)
$$

In Eq.10, range is the maximum step size set in the program. The updated velocity is projected to $[0,1]$ through Sigmoid, then the corresponding step size is obtained by multiply by a certain factor. After the velocity equation is improved, the position equation is still the same.

Double perturbation variation. In order to make the particles is more diverse, and enhance the particle's searching ability in the whole solution space, Gauss white noise and twice variation of genetics is introduced into the particle swarm algorithm. Gauss noise refers to the noise whose probability density function obeys Gauss distribution, and white noise refers to the noise whose power spectrum density obeys uniform distribution, then the Gauss white noise have the two characteristics at the same time. Variation is introduced from biological genetics, selecting some particles based on a certain probability to mutate [7].

In the process, each iteration, according to the probability of variation to select particles for the first perturbation variation, just like Eq.11.

$$
x_{i}=x_{i} \times\left(0.5+\sigma_{1}\right)
$$

In Eq.10, $x_{i}$ is the particle with its all dimensions which are selected according to the probability of variation, $\sigma_{1}$ is Gauss white noise random number, which has a Noise intensity of $1 \mathrm{dBW}$.

After each iteration of a certain algebra, the particle swarm is judged to see whether the algorithm is trapped in a local optimal value. If in, there is a second perturbation variation, namely a small perturbation variation of the global optimal solution, as shown in Eq.12.

$$
p g_{d_{1}, d_{2}}=p g_{d_{1}, d_{2}} \times\left(0.8+\sigma_{2}\right)
$$

In Eq.12, $p g$ is the global optimal solution, $p g_{d_{1}, d_{2}}$ are the two dimension randomly selected of the $p g, \sigma_{2}$ Gauss white noise random number with a Noise intensity of 0dBW.

After two Gauss white noise disturbance, particle may be beyond the solution space, then cross-border particle need to be modified, that is to produce a new particle in the solution space to improve the algorithm's global search ability.

Algorithm Flow. In the whole process, adjacency matrix is used to determine whether nodes are connected, and then the forward-backward sweep method is adopted in power flow calculation. The total algorithm process is shown in Fig.1.

\section{Analysis of example}

IEEE33 node network, such as Fig.2, is adopted for simulation analysis. The dotted line represents switch normally opening. Rated voltage is $12.66 \mathrm{kv}$, and the reference power is $10 \mathrm{MVA}$. The total load of this system is $3715 \mathrm{~kW}+\mathrm{j} 2300 \mathrm{KV}$ ar, and 4 distributed generations are set to access to the network. The initial population is 50 particles, and iteration time is $100 . c_{1}=1.5, c_{2}=2.5, w_{\text {ini }}=1.2, w_{\text {end }}=0.3$

$$
\text { , } p_{m 1}=0.4 \text { 。 }
$$

The result after optimized is shown in Tab.1. After optimization that network loss of PSO decreased $0.0116(56.8 \%)$, and the lowest node network voltage is increased by 0.0302 or $3.3 \%$. The network loss of improved PSO decreased 0.0134(65.7\%), and the lowest node network voltage is up to 0.9569, 
increased by $0.0441,4.8 \%$. So the feasibility of the algorithm is proved, and it is effective in reducing the network loss and improving the node voltage.

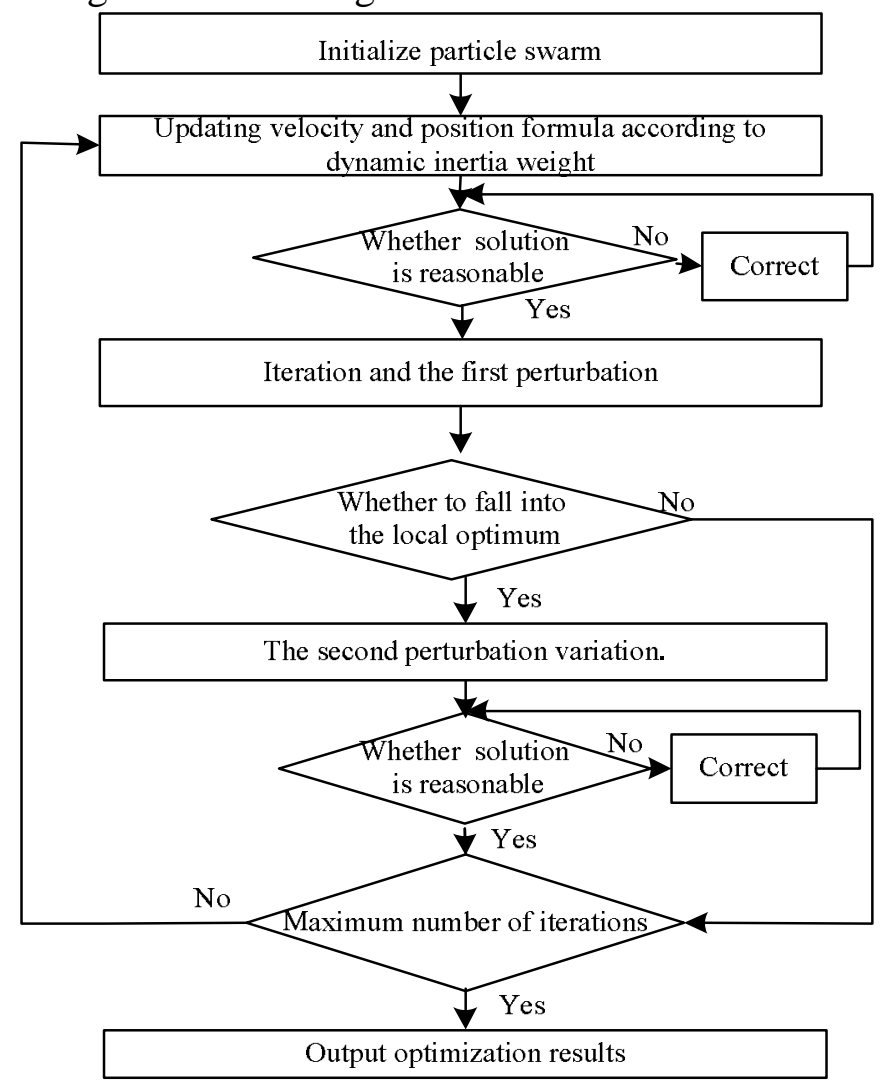

Fig.1 Flow chart of double perturbation mutation particle swarm optimization algorithm

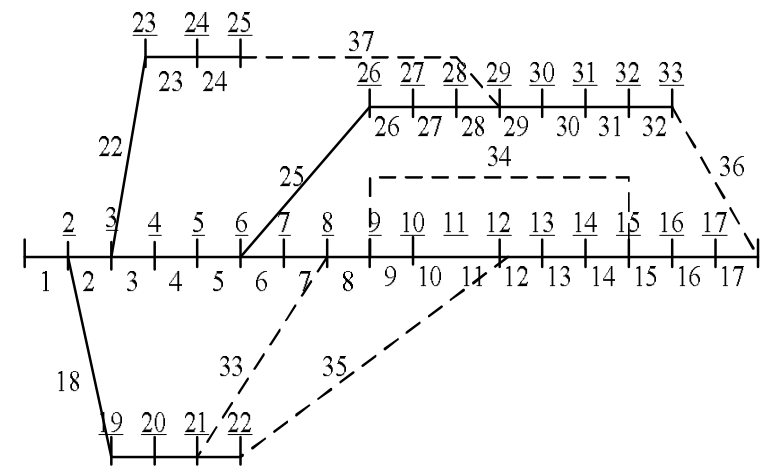

Fig.2 IEEE33 node distribution network

Tab.1 Result Comparison Analysis

\begin{tabular}{ccccc}
\hline Algorithm & $\begin{array}{c}\text { Position of } \\
\text { DG }\end{array}$ & $\begin{array}{c}\text { Series number } \\
\text { of the open } \\
\text { branch }\end{array}$ & $\begin{array}{c}\text { Network } \\
\text { loss } \\
\text { [p.u.] }\end{array}$ & $\begin{array}{c}\text { Minimum } \\
\text { node voltage } \\
\text { [p.u.] }\end{array}$ \\
\hline Before & - & $33,34,35,36,37$ & 0.0204 & 0.9128 \\
After PSO & $5,22,32,29$ & $11,32,34,37,6$ & 0.0088 & 0.9430 \\
After improved PSO & $29,31,15,30$ & $13,37,9,32,7$ & 0.0070 & 0.9569 \\
\hline
\end{tabular}

Network node voltage distribution is shown in Fig.3, it's obvious that node voltage rise after the optimization. Improved PSO is better and the node voltage which distributed power access to has been improved. Fig. 4 shows that the introduction of Gauss white noise perturbation mutation particle swarm algorithm is more quickly jump out of local optimum than the basic particle swarm algorithm, 
further verifying the feasibility of introducing Gauss white noise perturbation variations into the particle swarm optimization algorithm.

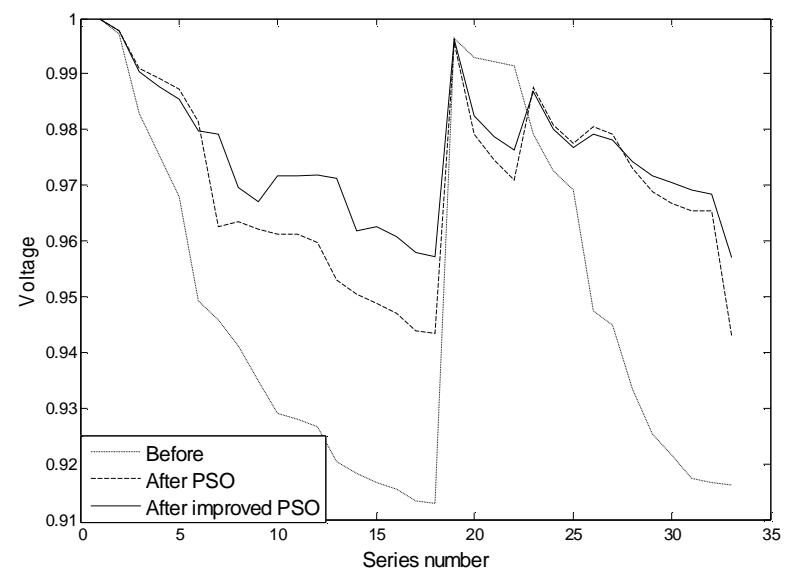

Fig.3 Node voltage comparison

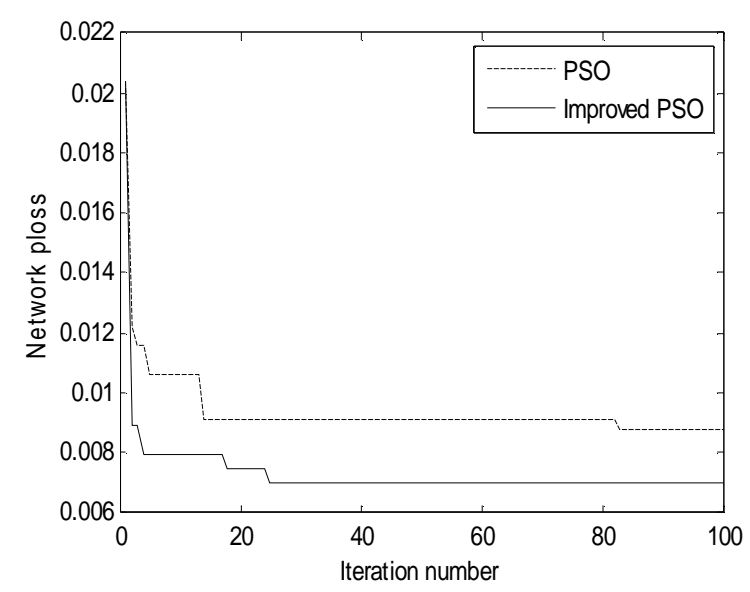

Fig.4 Comparison of iteration number

\section{Conclusion}

1. The rapid rise of new energy makes it very meaningful to consider accessing distributed generation in distribution network reconfiguration. The integer encoding is used in the reconstruction. The former $m$ dimension corresponds to the position of DG, and the posterior $n$ dimension corresponds to the disconnected branch.

2. The basic particle swarm algorithm is easy to fall into premature convergence, so this paper introduces the Gauss white noise perturbation variation, and the dynamic inertia weight and sigmoid function for the encoding into PSO. Besides, the speed formula is improved to adapt to the coding style. All these furtherly improves the overall and local search ability.

3. Through the analysis of simulation results, the feasibility of the algorithm has been verified. Either the access of distributed generation or network reconfiguration can reduce network loss and improve voltage, but effect of the two going at the same time, will be better.

\section{Reference}

[1] S. Hamidreza Alemohammad; Elahe Mashhour; Mohsen Saniei: Vol. 125 (2015), p. 15-22.

[2] LI Fei, QIU Zheng-mei, MA Li, and ZHU Tie-ming: Power System and Clean Energy, Vol. 27 (2011), p. 76-79. In Chinese.

[3] DONG Ru-Bang, YANG Qiang, and YAN Wen-jun:.Journal of Zhejiang University (Engineering Science), Vol. 49 (2015), p. 1982-1989. In Chinese.

[4] SUN Yan, MA Da-wei, WEI Hui: Electrical Measurement \& lnstrumentatinn, Vol. 50 (2013), p. 58-62. In Chinese.

[5] Hamid F, Mahmood-Reza H: ACO Based Algorihtm for Distributed Generation Sources Allocation and Sizing in Distribution system, in:2007 IEEE Power Tech. Lausanne(2007), p.555-560.

[6] AO Yong-cai, SHI Yi-bing, ZHANG Wei, and LI Yan-jun: .Journal of University of Electronic Science and Technology of China, Vol. 43 (2014), p. 874-880. In Chinese.

[7] GUO Guangsong, WANG Yanfang: Computer Engineering, Vol. 41 (2015), p. 182-189. In Chinese. 